Acta Crystallographica Section C

Crystal Structure

Communications

ISSN 0108-2701

\section{$N$-(tert-Butoxycarbonyl)-O-allyl- L-seryl-a-aminoisobutyryl-L-valine methyl ester: a protected tripeptide with an allylated serine residue}

\section{Hadgu Girmay Gebreslasie, ${ }^{\mathrm{a}, \mathrm{b}} \neq \varnothing$ Øyvind Jacobsen ${ }^{\mathrm{c}} \ddagger$ and Carl Henrik Görbitz ${ }^{\text {** }}$}

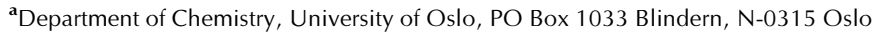
Norway, ${ }^{\mathbf{b}}$ Department of Medicine-Medical Biochemistry, College of Health Sciences, Mekelle University, PO Box 1871, Mekelle-Tigray, Ethiopia, and 'School of Pharmacy, University of Oslo, PO Box 1068 Blindern, N-0316 Oslo, Norway Correspondence e-mail: c.h.gorbitz@kjemi.uio.no

Received 23 June 2011

Accepted 22 July 2011

Online 6 August 2011

The title compound [systematic name $(6 S, 12 S)$-methyl 6-(allyloxymethyl)-12-isopropyl-2,2,9,9-tetramethyl-4,7,10-trioxo-3-oxa-5,8,11-triazatridecan-13-oate], $\mathrm{C}_{21} \mathrm{H}_{37} \mathrm{~N}_{3} \mathrm{O}_{7}$, containing the little studied $O$-allyl-L-serine residue [Ser(All)], crystallizes in the monoclinic space group $C 2$ with one molecule in the asymmetric unit. The compound is an analogue of the Ser140-Val142 segment of the water channel aquaporin-4 (AQP4). It forms a distorted type-II $\beta$-turn with a $P_{\mathrm{II}}-3_{10 L}-P_{\mathrm{II}}$ backbone conformation ( $P_{\mathrm{II}}$ is polyproline $\left.\mathrm{II}\right)$. The overall backbone conformation is markedly different from that of the $\mathrm{CO}$ (Pro139)-Val142 stretch of rat AQP4, but is quite similar to the corresponding segment of human AQP4, despite significant differences at the level of the individual residues. The side chain of the Ser(All) residue adopts a gauche conformation relative to the backbone $\mathrm{CO}-\mathrm{C}^{\alpha}$ and $\mathrm{C}^{\alpha}-\mathrm{N}$ bonds. The $\mathrm{H}$ atoms of the two $\mathrm{CH}_{2}$ groups in the Ser(All) side chain are almost eclipsed. The crystal packing of the title compound is divided into one-molecule-thick layers, each layer having a hydrophilic core and distinct hydrophobic interfaces on either side.

\section{Comment}

The title peptide, (I), was prepared as part of an ongoing effort (Jacobsen et al., 2009) to synthesize analogues of the Pro138Gly144 segment of the extracellular loop $\mathrm{C}$ of the water channel aquaporin-4 (AQP4) (Hasegawa et al., 1994; Jung et al., 1994; Hiroaki et al., 2006), which has emerged as an important target for the treatment of brain oedema (Nielsen et al., 1997; Manley et al., 2000; Amiry-Moghaddam et al., 2003; Amiry-Moghaddam \& Ottersen, 2003). The residues Pro139 and Val142 are thought to mediate adhesive interactions

$\ddagger$ These authors contributed equally to this work. between AQP4 molecules in contiguous cell membranes (Hiroaki et al., 2006; Engel et al., 2008; Tani et al., 2009). The electron diffraction structure of rat AQP4 (rAQP4) revealed that the segment Ser140-Gly144 forms a short $310^{\text {-helix }}$ [Protein Data Bank (PDB) code 2D57 (Hiroaki et al., 2006) and PDB code 2ZZ9 (Tani et al., 2009)]. We believe that compounds structurally mimicking a loop $\mathrm{C}$ segment containing at least one of the residues mediating adhesion can potentially have affinity for AQP4 and serve as lead compounds for the development of selective AQP4 ligands and, eventually, AQP4 inhibitors. (I) may be regarded as an analogue of the Ser140-Val141-Val142 segment of loop C, where the Ser residue has been allylated and Val141 has been (conservatively) substituted with another, structurally related, hydrophobic residue. The crystal structure of (I) renders a comparison with the backbone conformation of the AQP4 Ser140-Val142 segment possible. We have previously reported the crystal structure of a dipeptide analogue, Boc-Val-ValOMe (Boc = tert-butoxycarbonyl), of the Val141-Val142 segment of AQP4 (Jacobsen et al., 2011).<smiles>C=CCOC[C@H](NC(=O)OC(C)(C)C)C(=O)NC(C)(C)C(=O)N[C@H](C(=O)OC)C(C)C</smiles>

(I)

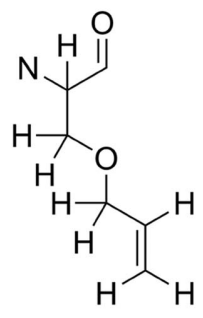

(II)

Regardless of the relationship between (I) and loop $\mathrm{C}$ of AQP4, the presence of two non-proteinogenic residues in (I), i.e. the synthetic residue $O$-allyl-L-serine $[\operatorname{Ser}(\mathrm{All})]$ and the naturally occurring achiral $\alpha, \alpha$-disubstituted residue $\alpha$-aminoisobutyric acid (Aib), makes the crystal structure of (I) potentially interesting per se. The Aib residue is found in a large number of antibiotic peptides produced by fungi known as peptaibiotics (Degenkolb \& Brückner, 2008). It is a conformationally restricted residue that preferentially adopts a $310^{-}$or $\alpha$-helical conformation (Ramachandran \& Chandrasekaran, 1972; Marshall \& Bosshard, 1972; Venkatraman et al., 2001; Aravinda et al., 2008). Compared to Aib, the properties of the Ser(All) residue have been less studied. As of 31 July 2011, only 170 compounds (including non-peptidic compounds) containing the $O$-allyl-L-serine fragment, (II), have been assigned a Chemical Abstracts Service Registry Number (American Chemical Society, 2008).

In many cases, the purpose of incorporating one or more Ser(All) residues in a peptide sequence has been to synthesize conformationally constrained peptides by ring-closing olefin 
metathesis (RCM) (Blackwell \& Grubbs, 1998; Blackwell et al., 2001; Hanessian et al., 2006; Jacobsen et al., 2009; Yamagata et al., 2011). The structural and pharmacological effects of RCM in peptides have recently been reviewed (Jacobsen et al., 2010). A small number of crystal structures have been obtained of the resulting cyclic peptides or hydrogenated versions thereof (Blackwell \& Grubbs, 1998; Hanessian et al., 2006; Boal et al., 2007; Abell et al., 2009; Yamagata et al., 2011). Perhaps because the properties of the cyclic peptides obtained after RCM have constituted the primary focus of several of the studies for which Ser(All)-containing peptides have been synthesized, only a single crystal structure, as far as we have been able to establish, has been reported of a peptide containing the $O$-allyl-L-serine residue (Boal et al., 2007). To determine the structural effect of $i \rightarrow i+3$ side-chain-to-sidechain RCM in the context of a predominantly $3_{10}$-helical peptide, Boal et al. (2007) determined the crystal structure of an Aib-rich octapeptide, Boc-Aib-Aib-Aib-Ser(All)-Aib-AibSer(All)-Aib-OMe, (III), before and after RCM. Similarly to (I), the $O$-allyl-L-serine residue in (III) is succeeded by an Aib residue.

So far, no crystal structures of peptides containing the closely related residues $O$-allyl-L-threonine or $S$-allyl-L-cysteine have been reported.

A turn is defined as a segment of a peptide which reverses the direction of the backbone (Venkatachalam, 1968; Rose et al., 1985). A $\beta$-turn is a turn comprising four residues which may, or may not, be stabilized by an intramolecular $i \rightarrow i+3$ hydrogen bond (Venkatachalam, 1968; Lewis et al., 1973; Richardson, 1981; Rose et al., 1985; Hutchinson \& Thornton, 1994; Guruprasad \& Rajkumar, 2000). The backbone of (I) changes direction by approximately $180^{\circ}$ from the quaternary carbon (C1) of the Boc group to the $\mathrm{C}^{\alpha}$-atom (C16) of the Val residue, thus constituting a $\beta$-turn (Fig. 1a). The distance between the two $\mathrm{C}$ atoms is 5.908 (5) $\AA$. In contrast to most $\beta$-turn subtypes, which feature an $i \rightarrow i+3$ intramolecular hydrogen bond, (I) does not form any intramolecular hydrogen bonds. The $\mathrm{H}$... O distance between the carbonyl $\mathrm{O}$ atom of the Boc group and the $\mathrm{NH}$ group of the Val residue is, however, relatively short at $3.34 \AA$ [ $\mathrm{N}$. . O = 3.898 (3) $\AA$ ] . As a result of the turn, the shape of the peptide may be characterized as an (open) disc with a protruding Ser(All) side chain.

It is well established that Aib can promote/stabilize helical conformations when incorporated in peptides (Burgess \& Leach, 1973; Karle \& Balaram, 1990; Marshall et al., 1990). However, no such effect is evident in the crystal structure of $(\mathrm{I})$. Instead the two chiral residues adopt polyproline II $\left(P_{\mathrm{II}}\right)$ conformations, with $(\varphi, \psi)$ of $\operatorname{Ser}($ All $)$ and Val being $\left[-59.0(4), 159.2(3)^{\circ}\right]$ and $\left[-70.3(3), 143.5(3)^{\circ}\right]$, respectively. The $P_{\mathrm{II}}$ conformation is believed to be an important local conformation of 'unfolded' peptides (Shi et al., 2006; Makowska et al., 2006). Because it lacks a stereogenic centre, the Aib residue can adopt left- or right-handed helical conformations with equal probability when incorporated in achiral peptides. However, when it is involved in intramolecular hydrogen bonding in a helical peptide containing
L-amino acids it preferentially adopts right-handed helical conformations (Aravinda et al., 2008). The central Aib residue in the nonhelical peptide (I) adopts a left-handed $33_{10}$-helical $\left(3_{10 L}\right)$ conformation, with $(\varphi, \psi)$ angles of [58.7 (4), $\left.33.1(4)^{\circ}\right]$. This conformation allows the formation of favourable intramolecular hydrophobic and van der Waals interactions between the tert-butyl and isopropyl groups and between the Ser(All) side chain and the Aib side chains. The backbone conformation of (I) closely resembles the conformations of several other fully protected tripeptides with a centrally placed Aib residue, e.g. Boc-L-Phe-Aib-L-Ile-OMe (Das et al., 2005), Boc-L-Ile-Aib-L-Val-OMe (Dutt et al., 2007), Boc-LAla-Aib-L-Val-OMe (Maji et al., 2004), Boc-L-Ala-Aib-L-IleOMe (Maji et al., 2004) and Boc-L-Ala-Aib-L-Ala-OMe, (IV) (Bosch et al., 1984). (IV) crystallizes in the space group $P 2_{1}$ and forms a distorted $\beta$-turn with a $P_{\mathrm{II}}-3_{10 L}-P_{\mathrm{II}}$ backbone conformation very similar to that of (I) (Fig. 1b). Recent conformational studies of (IV) by NMR, IR, vibrational circular dichroism (VCD) and electronic circular dichroism (ECD) spectroscopy, however, suggest that the structure of (IV) is more complex in aqueous solution than in the solid state (Schweitzer-Stenner et al., 2007). In contrast to the crystal structure, the population of Aib residues adopting a right-handed $3_{10}$-helical conformation appears to be greater

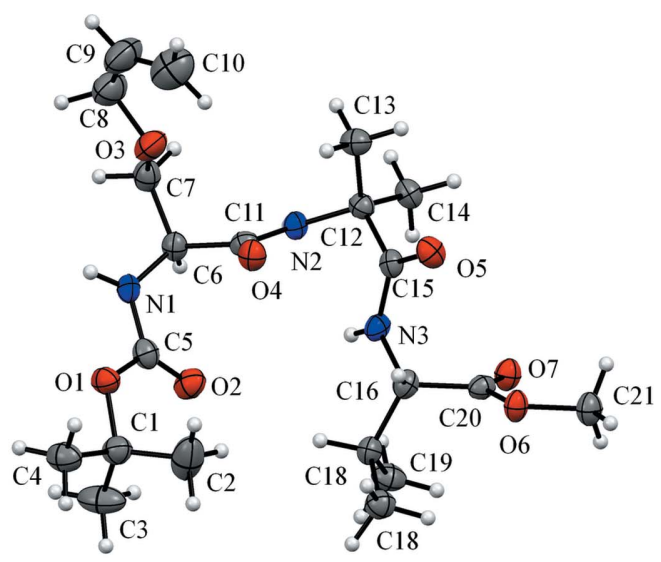

(a)

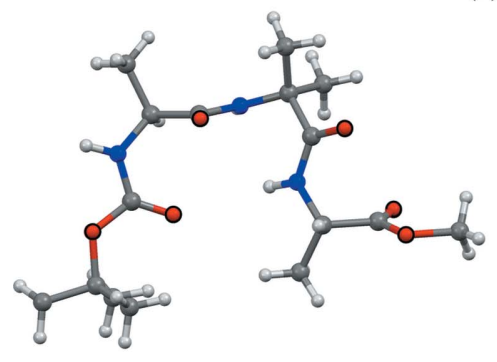

(b)

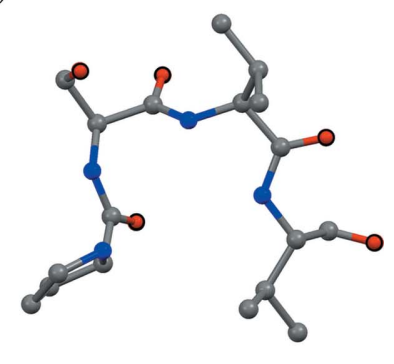

(c)
Figure 1

(a) The asymmetric unit of (I), with the atom numbering indicated. Displacement ellipsoids are drawn at the $50 \%$ probability level and $\mathrm{H}$ atoms are shown as spheres of arbitrary size. Bond lengths are normal, except that the $\mathrm{O} 3-\mathrm{C} 8$ and $\mathrm{C} 9-\mathrm{C} 10$ bonds in the allyl group are comparatively short at 1.395 (5) and 1.274 (7) $\AA$, respectively. (b) The structure of Boc-L-Ala-Aib-L-Ala-OMe, (IV) [Cambridge Structural Database (Allen, 2002) refcode COLSOL; Bosch et al., 1984]. (c) The Pro139-Val142 segment of hAQP4 (PDB code 3GD8; Ho et al., 2009). 


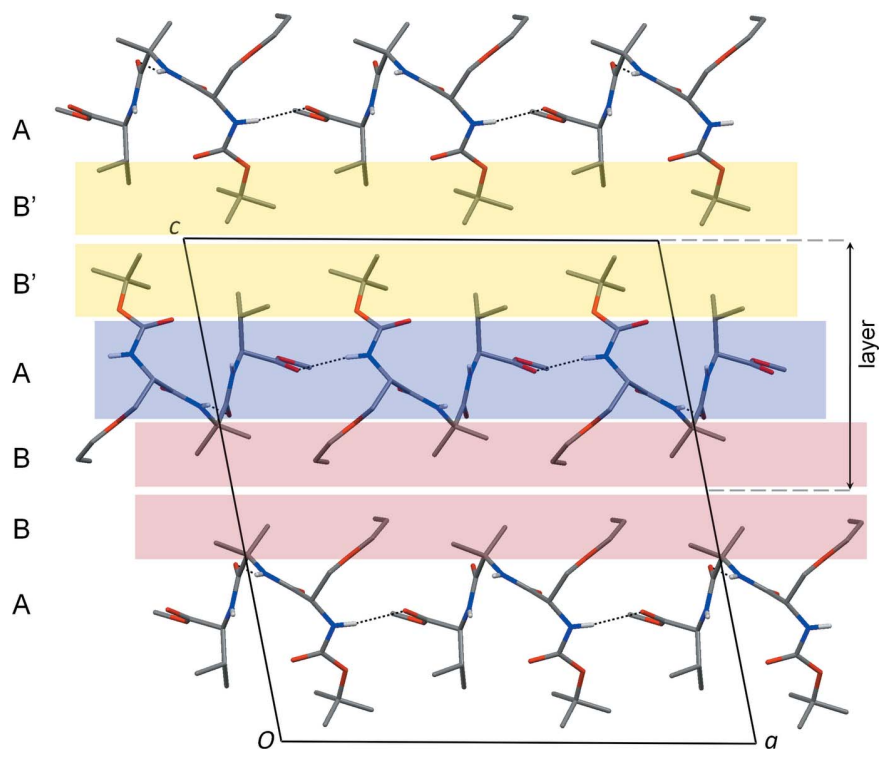

Figure 2

The unit cell and crystal packing of (I), viewed along the $b$ axis. $\mathrm{H}$ atoms bonded to $\mathrm{C}$ atoms have been omitted for clarity and hydrogen bonds are shown as dotted lines. The structure is divided into layers, limited by grey dashed lines, where each layer has a $B$ (red in the electronic version of the paper) $-A$ (blue) $-B^{\prime}$ (yellow) composition. Hydrogen bonds in the hydrophilic core $A$ are shown as dotted lines.

than the population of Aibs adopting a left-handed conformation, but it is difficult to predict whether this would also be the case for (I) in aqueous solution.

Based on the observed $(\varphi, \psi)$ angles of the Ser(All), Ala (residue $i+1$ ) and Aib (residue $i+2$ ), the structures of (I) and (IV) do not fall naturally into any of the standard $\beta$-turn classes (Richardson, 1981; Rose et al., 1985; Hutchinson \& Thornton, 1994; Guruprasad \& Rajkumar, 2000). The most similar type, the type-II $\beta$-turn, has dihedral angles $\left(\varphi_{i+1}\right.$, $\left.\psi_{i+1}\right)=\left(-60,120^{\circ}\right)$ and $\left(\varphi_{i+2}, \psi_{i+2}\right)=\left(80,0^{\circ}\right)($ Guruprasad \& Rajkumar, 2000).

Compared with the CO(Pro139)-Ser140-Val141-Val142 segment of rAQP4, (I) adopts a completely different overall conformation, as well as at the level of the individual residues (Table 1). While the Ser(All) and Aib residues of (I) adopt $P_{\mathrm{II}}$ and left-handed $3_{10}$-helical conformations, respectively, Ser140 of rAQP4 contribute to forming a short right-handed $3_{10}$-helix. In contrast, despite very different individual torsion angles, the overall backbone conformation of the $\mathrm{CO}$ (Pro139)Ser140-Val141-Val142 segment of human AQP4 (hAQP4) [Protein Data Bank (PDB) code 3GD8; Ho et al., 2009] (Fig. 1c) is quite similar to that of (I). One notable difference is that the central Ser140-Val141 peptide bond is flipped; (I) may be characterized as a distorted type-II $\beta$-turn, while the hAQP4 fragment has a distorted type-I $\beta$-turn conformation.

The side chain of the Ser(All) residue adopts a gauche conformation relative to both backbone bonds [N1- C6$\mathrm{C} 7-\mathrm{O} 3=69.5(3)^{\circ}$ and $\left.\mathrm{C} 11-\mathrm{C} 6-\mathrm{C} 7-\mathrm{O} 3=-51.9(3)^{\circ}\right]$. The same conformation is observed for one of the Ser(All) residues in the crystal structure of the octapeptide (III) (Boal et al., 2007), which may be stabilized by favourable $\sigma_{\mathrm{C} \alpha-\mathrm{H}} \rightarrow$ $\sigma^{*} \mathrm{C}-\mathrm{O}$ and $\sigma_{\mathrm{C} \beta-\mathrm{H}} \rightarrow \sigma^{*}{ }_{\mathrm{C}-\mathrm{N}}$ hyperconjugative interactions. On

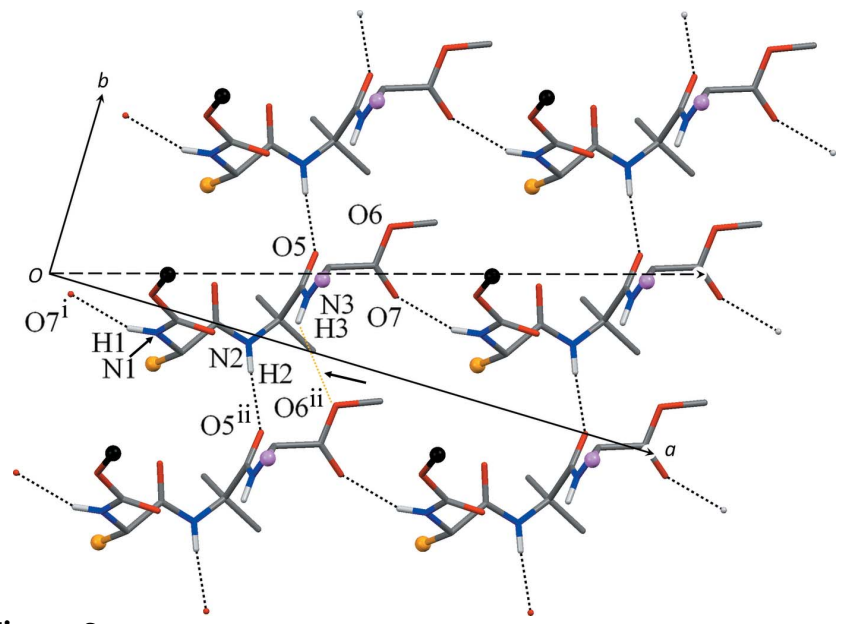

Figure 3

The hydrogen-bonding pattern in the $a b$ plane as viewed along $c^{*}$ with the directions of the $a$ and $b$ axes as well as the $a b$ diagonal (dashed line) indicated. The acceptor atoms are $\mathrm{O}^{\mathrm{i}}$ and $\mathrm{O} 5^{\mathrm{ii}}$ [symmetry codes: (i) $x-\frac{1}{2}$, $y-\frac{1}{2}, z$; (ii) $\left.x, y-1, z\right]$. A single long $\mathrm{N} 3-\mathrm{H} 3 \cdots \mathrm{O}^{\mathrm{ii}}$ contact (see Table 2; orange in the electronic version of the paper) is indicated with an arrow. [Also in the electronic version of the paper, the tert-butyl group, the Ser(All) side chain and the Val chain are depicted as black, orange and violet spheres, respectively.]

the other hand, the $\mathrm{C}^{\beta}-\mathrm{O}$ bond in the side chain of the second Ser(All) in (III) is positioned anti relative to the $\mathrm{CO}-\mathrm{C}^{\alpha}$ bond and gauche relative to the $\mathrm{C}^{\alpha}-\mathrm{N}$ bond, suggesting that the energy difference between the two side-chain conformers could be small. Going further out in the side chain, the $\mathrm{H}$ atoms of the two $\mathrm{CH}_{2}$ groups are almost eclipsed in all three cases. At first sight, this would appear to be an unfavourable conformation, but it should be noted that this arrangement reduces the steric repulsion between the $\mathrm{C}-\mathrm{H}$ groups and the oxygen lone pairs and furthermore allows some overlap between the four $\sigma_{\mathrm{C}-\mathrm{H}}$ orbitals and the two $\sigma^{*}{ }_{\mathrm{C}-\mathrm{O}}$ orbitals. Obviously, it is difficult, based on only three observations in two crystal structures, to draw any firm conclusions about the conformational preferences of the Ser(All) side chain without recourse to $a b$ initio or density functional theory (DFT) calculations, which is beyond the scope of the current study.

The crystal packing of (I) is divided into one-molecule-thick layers along the $c$ axis, each layer having a $B-A-B^{\prime}$ composition, where $A$ represents a hydrophilic core, and $B$ and $B^{\prime}$ are two different sets of hydrophobic groups (Fig. 2). In part $A$ the peptide backbones form two intermolecular hydrogen bonds that generate one $C(11)$ head-to-tail chain along the $a b$ diagonal and one $C(5)$ chain along the $b$ axis (Fig. 3); for graph-set notation, see Etter et al. (1990). Notably, the $\mathrm{N} 3-\mathrm{H} 3$ donor, which fails to form an intramolecular hydrogen bond, is only involved in a very weak interaction (Table 2). The hydrophobic part $B$ of each molecular layer comprises the Aib side chains and terminal Ser(All) olefin/ vinyl groups, while part $B^{\prime}$ has contributions from the isopropyl groups of the Val residues together with the tertbutyl groups. The overall $B-A-B^{\prime} \cdots B^{\prime}-A-B \cdots B-A-B^{\prime}$ stacking thus incorporates two distinct types of hydrophobic interfaces $B \cdots B$ and $B^{\prime} \cdots B^{\prime}$ parallel to the $a b$ plane, an arrangement that allows the vinyl groups to enjoy favourable 
$\pi-\pi$ interactions (Hunter \& Sanders, 1990) with each other. The internuclear distance between the partially positively charged terminal $\mathrm{H}$ atoms and the two $\mathrm{C}$ atoms in the $\pi$ system is on the order of 3.7-4.1

\section{Experimental}

The $N$-(tert-butoxycarbonyl)- $O$-allyl-L-serine building block was synthesized in an analogous manner to $\mathrm{N}$-(tert-butoxycarbonyl)- $\mathrm{O}$ benzyl-L-serine (Sugano \& Miyoshi, 1976) by double deprotonation of $\mathrm{N}$-(tert-butoxycarbonyl)-L-serine with $\mathrm{NaH}$ in dimethylformamide, followed by alkylation with allyl bromide (Jacobsen et al., 2009). The title compound, (I), was synthesized by standard solution-phase peptide coupling of $\mathrm{N}$-(tert-butoxycarbonyl)- $O$-allyl-L-serine and $\alpha$-aminoisobutyryl-L-valine methyl ester, which was generated in situ from $\alpha$-aminoisobutyryl-L-valine methyl ester trifluoroacetate by reaction with $N, N$-diisopropylethylamine. 1-Ethyl-3-(3-dimethylaminopropyl)carbodiimide (EDC) hydrochloride was used as coupling reagent and 1.0 equivalent of 1-hydroxybenzotriazole (HOBt) was added to catalyse the reaction and suppress epimerization (Jacobsen et al., 2009). The crude product was recrystallized twice from ethyl acetatehexane $(4: 1 v / v)$. About $5 \mathrm{mg}$ of (I) was dissolved in $30 \mu \mathrm{l}$ of ethyl acetate. Crystals appeared as water vapour diffused into the solution at room temperature.

\section{Crystal data}

$\mathrm{C}_{21} \mathrm{H}_{37} \mathrm{~N}_{3} \mathrm{O}_{7}$

$M_{r}=443.54$

Monoclinic, $C 2$

$a=19.753(4) \AA$

$b=5.9369(12) \AA$

$c=21.343(5) \AA$

$\beta=101.271(3)^{\circ}$

\section{Data collection \\ Bruker APEXII CCD diffractometer (SADABS; Bruker, 2007) \\ $T_{\min }=0.839, T_{\max }=0.979$ \\ Refinement \\ $R\left[F^{2}>2 \sigma\left(F^{2}\right)\right]=0.042$ \\ $w R\left(F^{2}\right)=0.107$ \\ $S=1.05$ \\ 2431 reflections \\ 280 parameters}

Absorption correction: multi-scan

$$
\begin{aligned}
& V=2454.7(9) \AA^{3} \\
& Z=4 \\
& \text { Mo } K \alpha \text { radiation } \\
& \mu=0.09 \mathrm{~mm}^{-1} \\
& T=105 \mathrm{~K} \\
& 0.60 \times 0.43 \times 0.24 \mathrm{~mm}
\end{aligned}
$$

\section{Table 1}

Main torsion angles $\left({ }^{\circ}\right)$ in the crystal structure of (I) and for the Ser-ValVal segments of three AQP4 structures from the PDB (identifier code given).

\begin{tabular}{lrrrr}
\hline Torsion angle $^{a}$ & This work & 2D $57^{b}$ & 2ZZ9 & 3GD8 $^{d}$ \\
\hline$\varphi_{1}$ & $-59.0(4)$ & -139.4 & -117.9 & -64.2 \\
$\psi_{1}$ & $159.2(3)$ & -7.8 & 5.6 & -21.4 \\
$\varphi_{2}$ & $58.7(4)$ & -59.0 & -67.9 & -118.2 \\
$\psi_{2}$ & $33.1(4)$ & -32.5 & -18.8 & -1.6 \\
$\varphi_{3}$ & $-70.3(3)$ & -30.6 & -21.7 & -50.9 \\
$\psi_{3}$ & $143.5(3)$ & -35.7 & -41.7 & -44.6 \\
\hline
\end{tabular}

Notes: (a) For (I), with reference to Fig. 1, the listed torsion angles are: $\varphi_{1}=\mathrm{C} 5-\mathrm{N} 1-$ $\mathrm{C} 6-\mathrm{C} 11, \psi_{1}=\mathrm{N} 1-\mathrm{C} 6-\mathrm{C} 11-\mathrm{N} 2, \varphi_{2}=\mathrm{C} 11-\mathrm{N} 2-\mathrm{C} 12-\mathrm{C} 15, \psi_{2}=\mathrm{N} 2-\mathrm{C} 12-\mathrm{C} 15-$ $\mathrm{N} 3, \varphi_{3}=\mathrm{C} 15-\mathrm{N} 3-\mathrm{C} 16-\mathrm{C} 20$ and $\psi_{3}=\mathrm{N} 3-\mathrm{C} 16-\mathrm{C} 20-\mathrm{O} 6 ;$; b ) Hiroaki et al. (2006); (c) Tani et al. (2009); (d) Ho et al. (2009).
Table 2

Hydrogen-bond geometry $\left(\AA{ }^{\circ}\right)$.

\begin{tabular}{lllll}
\hline$D-\mathrm{H} \cdots A$ & $D-\mathrm{H}$ & $\mathrm{H} \cdots A$ & $D \cdots A$ & $D-\mathrm{H} \cdots A$ \\
\hline $\mathrm{N} 1-\mathrm{H} 1 \cdots \mathrm{O}^{\mathrm{i}}$ & 0.88 & 2.23 & $3.060(3)$ & 157 \\
$\mathrm{~N} 2-\mathrm{H} 2 \cdots$ O5 $^{\mathrm{ii}}$ & 0.88 & 2.04 & $2.900(3)$ & 164 \\
$\mathrm{~N} 3-\mathrm{H} 3 \cdots 6^{\mathrm{ii}}$ & 0.88 & 2.95 & $3.621(3)$ & 135 \\
\hline
\end{tabular}

Symmetry codes: (i) $x-\frac{1}{2}, y-\frac{1}{2}, z$; (ii) $x, y-1, z$.

All $\mathrm{H}$ atoms were positioned with idealized geometry, with fixed $\mathrm{N}-\mathrm{H}=0.88 \AA$ and $\mathrm{C}-\mathrm{H}=0.95\left(s p^{2}\right), 0.98$ (methyl), 0.99 (methylene) or $1.00 \AA$ (methine), while permitting free rotation for the amino groups. $U_{\text {iso }}(\mathrm{H})$ values were set at $1.2 U_{\text {eq }}$ of the carrier atom or at $1.5 U_{\text {eq }}$ for methyl groups. In the absence of significant anomalous scattering effects, 1774 Friedel pairs were merged.

Data collection: APEX2 (Bruker, 2007); cell refinement: SAINTPlus (Bruker, 2007); data reduction: SAINT-Plus; program(s) used to solve structure: SHELXS97 (Sheldrick, 2008); program(s) used to refine structure: SHELXL97 (Sheldrick, 2008); molecular graphics: SHELXTL (Sheldrick, 2008); software used to prepare material for publication: SHELXTL.

Supplementary data for this paper are available from the IUCr electronic archives (Reference: SF3155). Services for accessing these data are described at the back of the journal.

\section{References}

Abell, A. D., Alexander, N. A., Aitken, S. G., Chen, H., Coxon, J. M., Jones, M. A., McNabb, S. B. \& Muscroft-Taylor, A. (2009). J. Org. Chem. 74, 43544356.

Allen, F. H. (2002). Acta Cryst. B58, 380-388.

American Chemical Society (2008). Chemical Abstracts Service. American Chemical Society, Columbus, OH, USA

Amiry-Moghaddam, M., Otsuka, T., Hurn, P. D., Traystman, R. J., Haug, F.-M., Froehner, S. C., Adams, M. E., Neely, J. D., Agre, P., Ottersen, O. P. \& Bhardwaj, A. (2003). Proc. Natl Acad. Sci. USA, 100, 2106-2111.

Amiry-Moghaddam, M. \& Ottersen, O. P. (2003). Nat. Rev. Neurosci. 4, 9911001.

Aravinda, S., Shamala, N. \& Balaram, P. (2008). Chem. Biodivers. 5, 1238 1262.

Blackwell, H. E. \& Grubbs, R. H. (1998). Angew. Chem. Int. Ed. 37, 3281-3284.

Blackwell, H. E., Sadowsky, J. D., Howard, R. J., Sampson, J. N., Chao, J. A., Steinmetz, W. E., O'Leary, D. J. \& Grubbs, R. H. (2001). J. Org. Chem. 66, 5291-5302.

Boal, A. K., Guryanov, I., Moretto, A., Crisma, M., Lanni, E. L., Toniolo, C., Grubbs, R. H. \& O'Leary, D. J. (2007). J. Am. Chem. Soc. 129, 6986-6987.

Bosch, R., Jung, G., Voges, K. P. \& Winter, W. (1984). Liebigs Ann. Chem. pp. 1117-1128.

Bruker (2007). APEX2, SAINT-Plus and SADABS. Bruker AXS Inc., Madison, Wisconsin, USA.

Burgess, A. W. \& Leach, S. J. (1973). Biopolymers, 12, 2599-2605.

Das, A. K., Banerjee, A., Drew, M. G. B., Ray, S., Haldar, D. \& Banerjee, A. (2005). Tetrahedron, 61, 5027-5036.

Degenkolb, T. \& Brückner, H. (2008). Chem. Biodivers. 5, 1817-1843.

Dutt, A., Dutta, A., Mondal, R., Spencer, E. C., Howard, J. A. K. \& Pramanik, A. (2007). Tetrahedron, 63, 10282-10289.

Engel, A., Fujiyoshi, Y., Gonen, T. \& Walz, T. (2008). Curr. Opin. Struct. Biol. 18, 229-235.

Etter, M. C., MacDonald, J. C. \& Bernstein, J. (1990). Acta Cryst. B46, 256-262. Guruprasad, K. \& Rajkumar, S. (2000). J. Biosci. 25, 143-156.

Hanessian, S., Yang, G., Rondeau, J.-M., Neumann, U., Betschart, C. \& Tintelnot-Blomley, M. (2006). J. Med. Chem. 49, 4544-4567.

Hasegawa, H., Ma, T., Skach, W., Matthay, M. A. \& Verkman, A. S. (1994). J. Biol. Chem. 269, 5497-5500.

Hiroaki, Y., Tani, K., Kamegawa, A., Gyobu, N., Nishikawa, K., Suzuki, H., Walz, T., Sasaki, S., Mitsuoka, K., Kimura, K., Mizoguchi, A. \& Fujiyoshi, Y. (2006). J. Mol. Biol. 355, 628-639. 
Ho, J. D., Yeh, R., Sandstrom, A., Chorny, I., Harries, W. E. C., Robbins, R. A., Miercke, L. J. W. \& Stroud, R. M. (2009). Proc. Natl Acad. Sci. USA, 106, 7437-7442.

Hunter, C. A. \& Sanders, J. K. M. (1990). J. Am. Chem. Soc. 112, 5525-5534.

Hutchinson, E. G. \& Thornton, J. M. (1994). Protein Sci. 3, 2207-2216.

Jacobsen, Ø., Gebreslasie, H. G., Klaveness, J., Rongved, P. \& Görbitz, C. H. (2011). Acta Cryst. C67, o278-o282.

Jacobsen, Ø., Klaveness, J., Ottersen, O. P., Amiry-Moghaddam, M. R. \& Rongved, P. (2009). Org. Biomol. Chem. 7, 1599-1611.

Jacobsen, Ø., Klaveness, J. \& Rongved, P. (2010). Molecules, 15, 6638-6677.

Jung, J. S., Bhat, R. V., Preston, G. M., Guggino, W. B., Baraban, J. M. \& Agre, P. (1994). Proc. Natl Acad. Sci. USA, 91, 13052-13056.

Karle, I. L. \& Balaram, P. (1990). Biochemistry, 29, 6747-6756.

Lewis, P. N., Momany, F. A. \& Scheraga, H. A. (1973). Biochim. Biophys. Acta, 303, 211-229.

Maji, S. K., Haldar, D., Drew, M. G. B., Banerjee, A., Das, A. K. \& Banerjee, A. (2004). Tetrahedron, 60, 3251-3259.

Makowska, J., Rodziewicz-Motowidlo, S., Baginska, K., Vila, J. A., Liwo, A., Chmurzynski, L. \& Scheraga, H. A. (2006). Proc. Natl Acad. Sci. USA, 103, 1744-1749.

Manley, G. T., Fujimura, M., Ma, T., Noshita, N., Filiz, F., Bollen, A. W., Chan, P. \& Verkman, A. S. (2000). Nat. Med. 6, 159-163.

Marshall, G. R. \& Bosshard, H. E. (1972). Circ. Res. 30-31, Suppl. II, 143-150.
Marshall, G. R., Hodgkin, E. E., Langs, D. A., Smith, G. D., Zabrocki, J. \& Leplawy, M. T. (1990). Proc. Natl Acad. Sci. USA, 87, 487-491.

Nielsen, S., Nagelhus, E. A., Amiry-Moghaddam, M., Bourque, C., Agre, P. \& Ottersen, O. P. (1997). J. Neurosci. 17, 171-180.

Ramachandran, G. N. \& Chandrasekaran, R. (1972). Progress in Peptide Research, edited by S. Lande, Vol. II (Proceedings of the Second American Peptide Symposium, Cleveland, 1970), p. 195. New York: Gordon \& Breach. Richardson, J. S. (1981). Adv. Protein Chem. 34, 167-339.

Rose, G. D., Gierasch, L. M. \& Smith, J. A. (1985). Adv. Protein Chem. 37, $1-109$.

Schweitzer-Stenner, R., Gonzales, W., Bourne, G. T., Feng, J. A. \& Marshall, G. R. (2007). J. Am. Chem. Soc. 129, 13095-13109.

Sheldrick, G. M. (2008). Acta Cryst. A64, 112-122.

Shi, Z., Chen, K., Liu, Z. \& Kallenbach, N. R. (2006). Chem. Rev. 106, $1877-$ 1897.

Sugano, H. \& Miyoshi, M. (1976). J. Org. Chem. 41, 2352-2353.

Tani, K., Mitsuma, T., Hiroaki, Y., Kamegawa, A., Nishikawa, K., Tanimura, Y. \& Fujiyoshi, Y. (2009). J. Mol. Biol. 389, 694-706.

Venkatachalam, C. M. (1968). Biopolymers, 6, 1425-1436.

Venkatraman, J., Shankaramma, S. C. \& Balaram, P. (2001). Chem. Rev. 101, 3131-3152.

Yamagata, N., Demizu, Y., Sato, Y., Doi, M., Tanaka, M., Nagasawa, K., Okuda, H. \& Kurihara, M. (2011). Tetrahedron Lett. 52, 798-801. 